\title{
Impacto de TI en las pequeñas y medianas empresas ¿es su efecto moderado por la intensidad de uso de TI de la industria?
}

\author{
Rodrigo Oliva ${ }^{1}$, Karina Carvajal ${ }^{*}$, Alejandro Cataldo ${ }^{2}$
}

\begin{abstract}
Title: IT impact on small and medium enterprises ¿is its effect moderate by intensity of IT use of industry?
This article aims to demonstrate that the impact of information technology (IT), on organizational performance of small and medium enterprises (SMEs), varies according to the intensity level of IT use of industry. For this we use microdata from the Second Longitudinal Survey of Business (ELE2), through which we established variables of intensity of IT use and organizational performance. Then, using X-Means we define indicators of intensity of IT use in SMEs and industries. The results showed that the intensity of IT use has positive effects on SMEs. Also, prove that the effects of the intensity of transactional IT use on performance, varies according to level of IT use of industry.
\end{abstract}

Keywords: Information technology; SMEs; IT impact; Organizational performance; Clustering.

Resumen: Este artículo tiene como objetivo demostrar que el impacto de las tecnologías de la información (TI), sobre el rendimiento de las pequeñas y medianas empresas (Pymes), varía según el nivel de intensidad de uso de TI de la industria. Para esto utilizamos microdatos provenientes de la Segunda Encuesta de Longitudinal de Empresas (ELE2), por medio de la cual establecimos variables de intensidad de uso de TI y de rendimiento organizacional. Luego, mediante X-Means definimos indicadores de intensidad de uso de TI en Pymes e industrias. Los resultados obtenidos demuestran que el uso de TI tiene efectos positivos en las Pymes. Además, prueban que los efectos del uso de TI transaccional sobre el rendimiento varían según el nivel de uso de la industria.

Palabras clave: Tecnologías de la información; Pymes; Impacto de TI; Rendimiento organizacional; Clustering.

Submitted: July $9^{\text {th }}, 2016 /$ Approved: May $10^{\text {th }}, 2018$

\section{Introducción}

En Chile el uso de tecnologías de la información (TI) ha pasado a ser parte de la cotidianidad de las personas. Chile, en tal sentido, destaca como líder latinoamericano en el uso de internet, telefonía móvil, ecommerce y e-government (Bilbao-Osorio, Crotti, Dutta, \& Lanvin, 2014; Mora et al., 2012). Aunque el progreso que las TI han traído es casi indiscutible a las sociedades, a nivel empresarial no se ha podido constatar que el uso de TI sea beneficioso para todo tipo de compañías, de hecho, a pesar del gran número de estudios sobre el impacto de las tecnologías en las empresas, estos no han logrado llegar a un consenso acerca de si las TI mejoran el rendimiento de las organizaciones o no. Esto es particularmente significativo para el caso de las pequeñas y medianas empresas (Pymes), cuyo acceso a las TI está más limitado que para las grandes empresas debido a sus restricciones de recursos (Mortenson \& Vidgen, 2016; Muñoz \& Pitti, 2016). De hecho, estudios muestran que las Pymes tienen dificultades para movilizar los recursos necesarios para hacer innovaciones (Enjolras, Camargo \& Schmitt, 2016). Este es un problema relevante para las naciones, especialmente las latinoamericanas, porque es sabido el gran aporte que este tipo de empresas realiza a las economías nacionales en términos de contratación de trabajadores (Correa \&Echavarría, 2013; Mora et al., 2012; Alves, Salvani, Bansi, Neto \& Galiba, 2016).
Con tal objetivo, realizamos un estudio sobre el impacto de las TI en las empresas más pequeñas. En general, nosotros buscamos comprobar que en las Pymes existe una relación positiva entre la intensidad de uso de TI y el rendimiento organizacional, pero esta relación es alterada por el tipo de industria. Para nuestro estudio usamos los datos de una encuesta nacional realizada en Chile (Segunda Encuesta Longitudinal de Empresas, ELE2), la cual es realizada periódicamente por el Gobierno de ese país.

El resto de este artículo está dividido en cuatro secciones. La siguiente resume los aspectos teóricos del impacto de TI, enfocándose especialmente en los trabajos locales hechos en esta área. La tercera sección describe la metodología utilizada. La cuarta sección presenta los resultados más relevantes obtenidos en el estudio. Finalmente, la quinta sección expone las conclusiones de la investigación realizada.

\section{Análisis teórico}

En esta sección, se exponen las bases teóricas de este trabajo, las que serán resumidas en dos subsecciones. La primera, describe la controversia que existe en la literatura de TI, sintetizando las distintas opiniones sobre la importancia de las tecnologías en las empresas. La segunda subsección resume los estudios sobre el impacto de las TI en las empresas chilenas.

(1) Departamento de Ingeniería Informática y Ciencias de la Computación, Universidad de Atacama. Copiapó, Chile.

(2) Escuela de Ingeniería Informática Empresarial, Universidad de Talca, Talca, Chile.

Autor de correspondencia: karina.carvajal@uda.cl 


\section{La importancia de las TI, un tema controversial}

El impacto de TI se ha convertido en un tema muy discutido en la literatura científica y profesional relacionada, siendo el artículo "IT doesn't matter" de Nicholas Carr (Carr, 2003) uno de los que ha causado mayor controversia desde su publicación. Este autor señaló que las tecnologías se han convertido en un commodity, por lo que no se puede considerar que otorguen valor estratégico a las empresas. Además, Carr plantea que, por lo tanto, a la hora de querer invertir en TI, las empresas debiesen concentrarse más en los riesgos que en los beneficios de las tecnologías, ya que los primeros pueden llevar a la compañía a caer en una desventaja de costos. Es por eso que (Carr, 2003), llama a las empresas a no ser líderes en inversión tecnológica, es decir, que sólo inviertan en tecnologías que ya hayan sido probadas por su competencia.

No obstante, tanto en la comunidad científica como profesional de TI, hubo opiniones dispares respecto a los dichos de Carr. DeJarnett, Laskey and Trainor (2004) describen el debate que se produjo entre los especialistas. Un argumento que se levantó en respuesta a Carr fue que la inversión en TI permite a las empresas obtener una ventaja temporal sobre su competencia, y el riesgo que conlleve esta inversión sólo superará a los beneficios, cuando estos riesgos no sean bien entendidos y/o gestionados. También los investigadores buscaron refutar las conclusiones de (Carr, 2003).

A pesar de lo influyente que resultó ser el artículo de Carr, él no fue el primero que realmente criticó el uso de TI en las empresas. Probablemente, la de Robert Solow, premio Nobel en economía, es una de las más fuertes críticas hechas al uso de TI en las compañías. En su momento, él declaró: "los computadores están en todos lados, excepto en las estadísticas de productividad”. Desde entonces, esta afirmación de Solow es conocida como "la paradoja de la productividad" (Brynjolfsson, 1993).

Aunque ha transcurrido una buena cantidad de tiempo, el debate sobre la paradoja de la productividad aun sigue vivo. Autores han postulado que los argumentos contra-TI fallan porque no innovar es un mal entendimiento de la naturaleza de la tecnología, ya que ésta es cambiante; por lo tanto, volverse seguidor TI, provocará que exista un retraso continuo que nunca permitirá acortar la brecha con los competidores más tecnologizados (Bannister \& Remenyi, 2005). En la misma línea argumental y más recientemente, Cataldo and McQueen (2014) presentaron la tesis que las TI pueden ser estratégicas en contextos competititivos con bajo nivel de uso tecnológico, en tal sentido, para ellos esta es una característica especial de los mercados locales en que compiten las PYMEs latinoamericanas. Por lo tanto, la incorporación de TI en Pymes sigue siendo una oportunidad estratégica para las más pequeñas empresas. Lo planteado por Cataldo and McQueen (2014) es consistente con estudios regionales que concluyen que existe una correlación entre el tamaño de las empresas y las Pymes (Guzman, Guzman \& Fuentes, 2015).

En suma, todo lo anterior muestra que el impacto de las TI en las empresas es un asunto controversial.

\section{Impacto de TI en Chile}

En Latinoamérica y en particular en Chile, el impacto de las TI en las empresas es aún una pregunta latente, principalmente por la insuficiente cantidad de trabajos en esta área. La escasez de estudios sobre el tema aumenta cuando se busca determinar el impacto de las TI en las Pymes, agravando la incerteza sobre el aporte que las nuevas tecnologías realmente hacen a las empresas. Sin perjuicio de lo anterior, existen algunos estudios relevantes que es importante mencionar.

Benavente, Lillo and Turén (2011) realizaron una investigación cualitativa/cuantitativa sobre el impacto de TI en las empresas chilenas. El estudio utilizó una muestra de 31 empresas, en donde la mayoría de éstas fueron Pymes. En la fase cualitativa, concluyeron que el 72,1\% de los directores de empresa, considera que el uso de TI mejora las ventas y que el 70,8\% que las TI aumentan las ganancias. En cambio, en la etapa cuantitativa, los autores encontraron que las empresas con mayores presupuestos de TI aumentaron sus costos, pero en el caso de las ventas y ganancias no se pudo especificar si estos habían mejorado.

Pero la controversia respecto al valor de las TI también es un tema controversial en Latinoamérica. Andrade Loaiza, Tucull Gallardo and González Garay (2010) investigaron cómo influye el uso de TI en las Pymes de la región de Magallanes (la provincia más austral de Chile), sus resultados mostraron que un importante porcentaje de Pymes $(47,7 \%)$ consideró que la inversión en TI hizo aumentar sus costos. En contraste, Concha, Burr and Suárez (2014), evaluando la percepción de las Pymes sobre el sistema de e-commerce "Chilecompra" (que es un market-place para las compras del Estado chileno), concluyeron que 46\% de las Pymes consideraba que el uso de este sitio disminuyó sus costos. Además, los autores encontraron que el 44\% de las Pymes declaraba que Chilecompra había aumentado sus ventas.

Los trabajos mencionados y otros tienen importantes limitaciones que impiden responder claramente si las TI afectan positivamente o no a las Pymes regionales. Una de las principales barreras para la generalización está vinculada al tipo y tamaño muestral usado en los estudios. Otra limitación tiene relación con los constructos y variables contextuales usadas, es decir, en cómo las TI son medidas y que factores organizacionales (inter o externos) son considerados en la investigación.

$\mathrm{Al}$ respecto, un aspecto poco explorado en sí, más que las tecnologías en forma agregada, es que ciertas combinaciones de TI son más beneficiosas que otras para las Pymes. En la literatura existen evidencias en tal sentido. Por ejemplo, Santoleri (2013) investigó el impacto de las TI sobre la innovación de productos en las empresas chilenas. El autor encontró que las empresas con mayor intensidad de uso de TI son las más propensas a innovar sus productos, pero también señaló que no toda combinación de tecnologías generaba beneficios para la organización. Esto último es consistente con Brynjolfsson (1993), quien concluyó que una mala gestión de TI o una inversión innecesaria, no creaba los beneficios esperados a la firma. Por lo tanto, cabe preguntarse si ¿existen combinaciones TI que generan mayores beneficios para las Pymes que otras? Es decir: 
H1: La intensidad de uso de TI influirá positivamente en el rendimiento organizacional de las Pymes.

Por otra parte, es sabido que los niveles de adopción de TI no son homogéneos entre industrias (Gandhi, Khanna, \& Ramaswamy, 2016). En otras palabras, en algunas industrias sus Pymes, en promedio, no hacen un uso intensivo de TI, en tales casos según (Cataldo \& McQueen, 2014), aquellas Pymes de estas industrias que utilizan TI más intensivamente que sus competidoras, tendrían mejores rendimientos debido a las brechas digitales que se generan entre ellas. No obstante, hay trabajos que señalan lo contrario. Por ejemplo Ramírez-Correa and Alfaro-Pérez (2011), indican que no existen diferencias en el rendimiento de las empresas que hacen mayor y menor inversión de TI. En contraste Reichert and Zawislak (2014), al investigar empresas de distintas industrias brasileras, infirieron que las compañías de las industrias que hicieron menor intensidad de uso de TI fueron las que obtuvieron un mayor rendimiento, en cambio las pertenecientes a industrias de mayores niveles de intensidad de uso obtuvieron un rendimiento inferior. Por lo tanto, lo anterior nos lleva a levantar una segunda pregunta: ¿el grado promedio de uso de TI en la industria influye de forma importante en los efectos que puedan producir las TI dentro de las Pymes? Esto nos permite plantear la segunda hipótesis de investigación:

H2: El nivel de intensidad de uso de TI de la industria, moderará la relación entre la intensidad de uso de TI de las Pymes y su rendimiento organizacional.

\section{Metodología}

Para la obtención de los datos utilizamos la ELE2 (disponible en http://www.economia.gob.cl), la cual es una encuesta nacional realizada por el Gobierno de Chile. Esta encuesta fue aplicada a 7.062 empresas chilenas de distintos tamaños e industrias. Dado que en esta investigación nos enfocamos en Pymes, filtramos las empresas por su tamaño, dejando organizaciones que poseen desde 6 a 250 trabajadores. Este primer filtrado redujo la muestra original a 4.142 casos.

El siguiente pre-procesamiento realizado fue eliminar los datos vacíos (missing) siguiendo la máxima de si un dato no existía, procurar salvar el caso completo infiriendo la respuesta según los otros datos, en caso contrario, eliminar el caso completo. Por lo que finalmente la muestra para este estudio fue de 3.661 casos. En la Tabla 1 se muestra la cantidad de Pymes por industria que posee la muestra final.

Tabla 1. Muestra final de Pymes distribuidas por industria.

\begin{tabular}{l|l|l}
\hline Industria & Descripción & Pymes \\
\hline A & Agricultura, ganadería, caza y silvicultura & 197 \\
\hline B & Pesca & 145 \\
\hline C & Explotación de minas y canteras & 134 \\
\hline D & Industria manufacturera & 572 \\
\hline E & Suministro de electricidad, gas y agua & 62 \\
\hline F & Construcción & 431 \\
\hline G & $\begin{array}{l}\text { Comercio al por mayor y menor, reparación de } \\
\text { vehículos automotores, motocicletas, efectos per- }\end{array}$ & 661 \\
\hline sonales y enseres domésticos & 406 \\
\hline I & Hoteles y restaurantes & 358 \\
\hline J & Transporte, almacenamiento y comunicaciones \\
\hline K & Intermediación financiera & 116 \\
\hline Octividades Inmobiliarias, empresariales y de & alquiler & 371 \\
\hline Otras actividades de servicios comunitarios, so- & 208 \\
\hline ciales y personales & 3.661 \\
\hline
\end{tabular}

Variables de intensidad de uso de TI y rendimiento organizacional

Para determinar las variables de intensidad de uso de TI y de rendimiento organizacional, se recurrió a la literatura previa, seleccionando como variables a aquellas más frecuentemente citadas y que fueran posibles obtenerlas a partir de la información disponible en la ELE2. Tales variables se muestran en la Tabla 2 y Tabla 3. 
Tabla 2. Lista de variables de intensidad de uso de TI seleccionadas.

\begin{tabular}{|c|c|c|}
\hline Variables de uso de TI & Descripción & Artículos revisados \\
\hline Computador personal (PC) & Uso de computadores de escritorio y/o portátiles. & $\begin{array}{l}\text { (Santoleri, 2013) } \\
\text { (Mora et al., 2012) } \\
\text { (Andrade Loaiza, Tucull Gallardo \& González Garay, 2010) } \\
\text { (Ramírez-Correa \& Alfaro-Pérez, 2011) }\end{array}$ \\
\hline Correo electrónico (e-mail) & Uso de correo electrónico. & $\begin{array}{l}\text { (Lohrke, McClure Franklin, \& Frownfelter-Lohrke, 2006) } \\
\text { (Mora et al., 2012) } \\
\text { (Brasini \& Freo, 2012) } \\
\text { (Andrade Loaiza, Tucull Gallardo \& González Garay, 2010) } \\
\text { (Trung Pham, 2010) }\end{array}$ \\
\hline Banca electrónica (e-banking) & Acceso a servicios financieros por medio de Internet. & $\begin{array}{l}\text { (Mora et al., 2012) } \\
\text { (Andrade Loaiza, Tucull Gallardo \& González Garay, 2010) }\end{array}$ \\
\hline Internet & Uso de internet. & $\begin{array}{l}\text { (Santoleri, 2013) } \\
\text { (Lohrke, McClure Franklin, \& Frownfelter-Lohrke, 2006) } \\
\text { (Mora et al., 2012) } \\
\text { (Brasini \& Freo, 2012) } \\
\text { (Andrade Loaiza, Tucull Gallardo \& González Garay, 2010) } \\
\text { (Trung Pham, 2010) } \\
\text { (Papastathopoulos \& Beneki, 2010) }\end{array}$ \\
\hline Intranet & Uso de Intranet. & \begin{tabular}{|l} 
(Santoleri, 2013) \\
(Brasini \& Freo, 2012) \\
(Andrade Loaiza, Tucull Gallardo \& González Garay, 2010) \\
(Trung Pham, 2010) \\
(Papastathopoulos \& Beneki, 2010)
\end{tabular} \\
\hline Red local (LAN/WLAN) & Uso de red de área local alámbrica o inalámbrica. & $\begin{array}{l}\text { (Brasini \& Freo, 2012) } \\
\text { (Andrade Loaiza, Tucull Gallardo \& González Garay, 2010) } \\
\text { (Trung Pham, 2010) } \\
\text { (Papastathopoulos \& Beneki, 2010) }\end{array}$ \\
\hline Software de administración & $\begin{array}{l}\text { Uso de software de administración como de contabilidad, finanzas, ERP, facturación y si- } \\
\text { milares. }\end{array}$ & $\begin{array}{l}\text { (Santoleri, 2013) } \\
\text { (Brasini \& Freo, 2012) } \\
\text { (Trung Pham, 2010) } \\
\text { (Papastathopoulos \& Beneki, 2010) } \\
\text { (Ramírez-Correa \& Alfaro-Pérez, 2011) }\end{array}$ \\
\hline Software de ventas y marketing & $\begin{array}{l}\text { Uso de software de ventas, marketing y/o gestión de clientes (Ej: CRM, puntos de venta, } \\
\text { control de cajas). }\end{array}$ & $\begin{array}{l}\text { (Santoleri, 2013) } \\
\text { (Sheng \& Mykytyn Jr., 2002) } \\
\text { (Trung Pham, 2010) } \\
\text { (Chang, Park, \& Chaiy, 2010) } \\
\text { (Ali, Habidin, Jamaludin, Khaidir, \& Shazali, 2013) } \\
\text { (Papastathopoulos \& Beneki, 2010) } \\
\text { (Ramírez-Correa \& Alfaro-Pérez, 2011) } \\
\end{array}$ \\
\hline Software específico & Uso de software específico al giro del negocio (EJ: Autocad, sistemas de reserva, entre otros). & \begin{tabular}{|l|} 
(Santoleri, 2013) \\
(Mora et al., 2012) \\
(Brasini \& Freo, 2012) \\
(Andrade Loaiza, Tucull Gallardo \& González Garay, 2010) \\
(Ramírez-Correa \& Alfaro-Pérez, 2011) \\
\end{tabular} \\
\hline $\begin{array}{l}\text { Software de logística y relación con } \\
\text { proveedores }\end{array}$ & $\begin{array}{l}\text { Uso de software de inventarios, logística y relación con proveedores (Ej: control de existen- } \\
\text { cias y de flota). }\end{array}$ & $\begin{array}{l}\text { (Brasini \& Freo, 2012) } \\
\text { (Trung Pham, 2010) } \\
\text { (Papastathopoulos \& Beneki, 2010) } \\
\text { (Ramírez-Correa \& Alfaro-Pérez, 2011) } \\
\end{array}$ \\
\hline Conocer empresa y productos & $\begin{array}{l}\text { El sitio web de la organización permite conocer detalles de la empresa y de sus productos } \\
\text { y servicios. }\end{array}$ & $\begin{array}{l}\text { (Lohrke, McClure Franklin, \& Frownfelter-Lohrke, 2006) } \\
\text { (Trung Pham, 2010) } \\
\text { (Papastathopoulos \& Beneki, 2010) }\end{array}$ \\
\hline Venta en línea con entrega fuera de línea & El sitio web empresarial permite vender en línea con entrega fuera de línea. & $\begin{array}{l}\text { (Santoleri, 2013) } \\
\text { (Concha, Burr \& Suárez, 2014) } \\
\text { (Mora et al., 2012) } \\
\text { (Papastathopoulos \& Beneki, 2010) } \\
\text { (Lohrke, McClure Franklin, \& Frownfelter-Lohrke, 2006) }\end{array}$ \\
\hline Venta y entrega en línea & $\begin{array}{l}\text { El sitio web de la organización permite vender en línea con entrega/descarga online de pro- } \\
\text { ductos (informe, software, música, otros). }\end{array}$ & $\begin{array}{l}\text { (Santoleri, 2013) } \\
\text { (Concha, Burr \& Suárez, 2014) } \\
\text { (Mora et al., 2012) } \\
\text { (Papastathopoulos \& Beneki, 2010) } \\
\text { (Lohrke, McClure Franklin, \& Frownfelter-Lohrke, 2006) }\end{array}$ \\
\hline Pago en línea (Web Pay) & El sitio web de la empresa permite pagar en línea. & $\begin{array}{l}\text { (Santoleri, 2013) } \\
\text { (Concha, Burr \& Suárez, 2014) } \\
\text { (Mora et al., 2012) } \\
\text { (Papastathopoulos \& Beneki, 2010) } \\
\text { (Lohrke, McClure Franklin, \& Frownfelter-Lohrke, 2006) } \\
\end{array}$ \\
\hline Información cuenta o factura & El sitio web de la compañía permite obtener información de la cuenta o facturación. & \begin{tabular}{|l|} 
(Concha, Burr \& Suárez, 2014) \\
(Papastathopoulos \& Beneki, 2010) \\
(Magal, Koslage, \& Levenburg, 2008) \\
(Lohrke, McClure Franklin, \& Frownfelter-Lohrke, 2006)
\end{tabular} \\
\hline Personalización sitio web & El sitio web organizacional se puede personalizar para clientes antiguos o frecuentes. & $\begin{array}{l}\text { (Lohrke, McClure Franklin, \& Frownfelter-Lohrke, 2006) } \\
\text { (Papastathopoulos \& Beneki, 2010) }\end{array}$ \\
\hline Seguimiento compra-venta & El sitio web de la empresa permite realizar seguimientos de compras o ventas. & $\begin{array}{l}\text { (Concha, Burr \& Suárez, 2014) } \\
\text { (Magal et al., 2008) } \\
\text { (Papastathopoulos \& Beneki, 2010) } \\
\text { (Lohrke, McClure Franklin, \& Frownfelter-Lohrke, 2006) }\end{array}$ \\
\hline Transacción con proveedores & El sitio web de la compañía permite a los proveedores realizar transacciones con ella. & $\begin{array}{l}\text { (Concha, Burr \& Suárez, 2014) } \\
\text { (Santoleri, 2013) } \\
\text { (Lohrke, McClure Franklin, \& Frownfelter-Lohrke, 2006) } \\
\text { (Papastathopoulos \& Beneki, 2010) }\end{array}$ \\
\hline
\end{tabular}


Tabla 3. Lista de variables de rendimiento organizacional seleccionadas.

\begin{tabular}{|c|c|c|}
\hline Variables rendimiento organizacional & Descripción & Artículos revisados \\
\hline Ventas & $\begin{array}{l}\text { Ingresos que obtiene la empresa tras llevar a cabo su ac- } \\
\text { tividad principal. }\end{array}$ & $\begin{array}{l}\text { (Richard, Devinney, Yip, \& Johnson, 2008) } \\
\text { (Concha, Burr \& Suárez, 2014) } \\
\text { (Benavente, Lillo \& Turén, 2011) } \\
\text { (Juárez, 2011) } \\
\text { (Reichert \& Zawislak, 2014) }\end{array}$ \\
\hline Costos & $\begin{array}{l}\text { Costos de la empresa tras llevar a cabo su actividad } \\
\text { principal. }\end{array}$ & $\begin{array}{l}\text { (Lohrke, McClure Franklin, \& Frownfelter-Lohrke, 2006) } \\
\text { (Concha, Burr \& Suárez, 2014) } \\
\text { (Benavente, Lillo \& Turén, 2011) } \\
\text { (Andrade Loaiza, Tucull Gallardo \& González Garay, 2010) } \\
\text { (Mora et al., 2012) }\end{array}$ \\
\hline Ganancias & Utilidades obtenidas por la empresa. & $\begin{array}{l}\text { (Richard, Devinney, Yip, \& Johnson, 2008) } \\
\text { (Benavente, Lillo \& Turén, 2011) } \\
\text { (Abu-Jarad, Yusof, \& Nikbin, 2010) } \\
\text { (Reichert \& Zawislak, 2014) }\end{array}$ \\
\hline Flujo de caja & Dinero en efectivo que maneja la empresa. & $\begin{array}{l}\text { (Richard, Devinney, Yip, \& Johnson, 2008) } \\
\text { (Hudson, Smart \& Bourne, 2001) } \\
\text { (Abu-Jarad, Yusof, \& Nikbin, 2010) }\end{array}$ \\
\hline Innovación de productos & $\begin{array}{l}\text { Mejora sustantiva o creado un nuevo producto en los } \\
\text { bienes/servicios que comercializa }\end{array}$ & $\begin{array}{l}\text { (Lohrke, McClure Franklin, \& Frownfelter-Lohrke, 2006) } \\
\text { (Santoleri, 2013) }\end{array}$ \\
\hline Innovación logística & $\begin{array}{l}\text { Realización de actividades vinculadas a la elaboración } \\
\text { e introducción de mejoras sustantivas o de nuevos } \\
\text { métodos de distribución (vinculados a la logística de } \\
\text { la empresa) }\end{array}$ & $\begin{array}{l}\text { (Lohrke, McClure Franklin, \& Frownfelter-Lohrke, 2006) } \\
\text { (Santoleri, 2013) }\end{array}$ \\
\hline Innovación marketing & $\begin{array}{l}\text { Desarrollo de nuevos métodos de comercialización } \\
\text { que impliquen cambios significativos del diseño o en- } \\
\text { vasado de un producto, su posicionamiento, su pro- } \\
\text { moción o su tarificación. }\end{array}$ & $\begin{array}{l}\text { (Lohrke, McClure Franklin, \& Frownfelter-Lohrke, 2006) } \\
\text { (Santoleri, 2013) }\end{array}$ \\
\hline Innovación organizacional & $\begin{array}{l}\text { Ejecución de actividades vinculadas a la elaboración } \\
\text { y la planificación de nuevos métodos de organización } \\
\text { o en la relación con otras empresas u organizaciones } \\
\text { relacionadas }\end{array}$ & $\begin{array}{l}\text { (Lohrke, McClure Franklin, \& Frownfelter-Lohrke, 2006) } \\
\text { (Santoleri, 2013) }\end{array}$ \\
\hline
\end{tabular}

Para facilitar la posterior creación de indicadores de intensidad de uso de TI para las empresas de la muestra, decidimos agrupar las variables de TI en base a la clasificación de (Aral \& Weill, 2007), la cual considera cuatro categorías de TI: infraestructura, transaccional, informacional y estratégica. Según estos autores, la categoría de infraestructura proporciona la base de la compartición servicios de TI (tanto técnicos como humanos, por ejemplo, servidores, redes, computadores, entre otros). Las TI transaccionales están relacionadas a la automatización de procesos. Las TI informacional proporcionan información para administración, contabilidad, informes y minería de datos. Las TI estratégicas apoyan la entrada en un nuevo mercado o el desarrollo de nuevos productos, servicios o procesos de negocios.

Como se puede observar en los resultados obtenidos, solo un tipo de software pudo ser categorizado como "estratégico" (Personalización sitio web), por lo que se decidió que las categorías "informacional" y "estratégica" fueran fusionadas, dado que ambas apoyan la toma de decisiones y la capacidad de respuesta de la compañía (Aral \& Weill, 2007). Por consiguiente, la clasificación de TI que utilizamos en este estudio es la que se muestra en la Tabla 4.

Tabla 4. Modificación de la clasificación de variables de TI de (Aral \& Weill, 2007).

\begin{tabular}{|c|c|c|}
\hline $\begin{array}{l}\text { PC } \\
\text { Internet } \\
\text { Intranet } \\
\text { LAN/WLAN } \\
\text { e-mail } \\
\text { Conocer empresa y productos }\end{array}$ & $\begin{array}{l}\text { e-banking } \\
\text { Software de ventas y marketing } \\
\text { Software específico } \\
\text { Venta en línea con entrega fuera de línea } \\
\text { Venta y entrega en línea } \\
\text { Web Pay } \\
\text { Transacción con proveedores }\end{array}$ & $\begin{array}{l}\text { Software de administración } \\
\text { Software de logística y relación con proveedores } \\
\text { Información cuenta o factura } \\
\text { Seguimiento compra-venta } \\
\text { Personalización sitio web }\end{array}$ \\
\hline
\end{tabular}




\section{Indicadores de intensidad de uso de TI}

Los estudios sobre TI, normalmente usan métodos lineales para poder establecer indicadores de intensidad de uso de TI en las empresas (Santoleri, 2013). Sin embargo, el uso de estos métodos puede llevar a una determinación errónea, ya que en estos modelos usualmente a cualquier tipo de TI se les da el mismo valor, pasando por alto que existen algunas tecnologías que son más complejas que otras, ya que requieren mayores conocimientos y recursos (por ejemplo, no da lo mismo que una organización cuente sólo con software ofimático y otra tenga sistemas como CRM).

Es dado lo anterior, que en esta investigación decidimos utilizar algoritmos de clustering para encontrar inductivamente niveles de intensidad de uso de TI. Este tipo de algoritmos ya ha sido usado en trabajos previos. Con propósitos similares, la mayoría de los investigadores han usado K-Means como algoritmo de minería de datos, a diferencia de ellos nosotros decidimos utilizar X-Means que es un algoritmo basado en K-Means pero que además es capaz de determinar automáticamente el número de clúster (Pelleg \& Moore, 2000). Siendo esto último beneficioso para este estudio, ya que evita que se predetermine el número de niveles de intensidad de uso a encontrar en la muestra. La ejecución del algoritmo X-Means se llevó a cabo por medio del software Rapidminer Studio.

Con el fin de poder evaluar la calidad de los clúster resultantes, usamos el índice Silhouette (Rousseeuw, 1987). Éste evalúa la distancia intra e inter clúster, siendo los conglomerados más compactos y más alejados de otros grupos los que representan una mayor calidad de clusterización. Además el índice Silhouette ha demostrado ser más exacto que otros índices de calidad (Fallahpour, Zadeh, \& Lakvan, 2014). Los resultados de Silhouette varían entre -1 y 1 , si el resultado es cercano a -1 significa que hubo una mala clusterización; si es cercano a 0 , los elementos clusterizados pudiesen pertenecer a otros clúster; por último, si el resultado es cercano a 1 implica que hubo una buena clusterización (Fallahpour, Zadeh, \& Lakvan, 2014). Por lo tanto, en este estudio establecimos que la clusterización sería de calidad cuando el Silhouette fuese mayor o igual a 0,5 .

Luego de clusterizar cada una de las tres categorías de TI (infraestructura, transaccional e informacional - estratégica), establecimos el nivel de intensidad de uso de cada empresa, según al clúster a que éstas perteneciesen. Esto nos permitió identificar qué clúster agrupaba a las empresas más tecnologizadas y, posteriormente, reconocer las industrias más o menos tecnologizadas.

\section{Nivel de intensidad de uso de la industria}

Una vez determinado el nivel de intensidad de uso de TI en cada empresa de la muestra, establecimos el nivel de intensidad de uso de TI en las industrias. En este estudio se entenderá que el nivel de intensidad de uso de TI en una industria es el uso promedio de TI que hacen todas las empresas pertenecientes a tal industria.

\section{Análisis inferencial}

Un último análisis fue la prueba de hipótesis. En el caso de H1, utilizamos Rho de Spearman para probar la relación entre intensidad de uso de TI y las variables de rendimiento usadas en este estudio. Para H2 utilizamos T-test que permite comparar los efectos producidos por las TI en industrias de distintos niveles de intensidad de uso.

Se usó un nivel de significancia $p=0,05$ para todas las pruebas.

\section{Resultados}

Esta sección está dividida en dos subsecciones. La primera presenta los resultados del análisis de clusterización en cada una de las tres categorías de TI. La segunda, muestra los resultados y análisis de las pruebas de hipótesis.

\section{Resultados clusterización}

En cada una de las tres clusterizaciones hechas (infraestructura, transaccionales e informacionales-estratégicas), el algoritmo X-Means encontró cuatro grupos. Como un primer comentario se pudo observar que cada uno de estos conglomerados representó distintos niveles de intensidad de uso de TI que hacen las Pymes. Cabe destacar, que cada uno de los clúster resultantes obtuvo un índice Silhouette sobre 0,5 ; lo que satisfacía nuestra condición inicial sobre la calidad de los clúster (Fallahpour, Zadeh, \& Lakvan, 2014). A continuación, se muestran los resultados de la clusterización para cada una de las tres categorías de TI.

\section{1) TI de infraestructura}

La clusterización de las variables de TI de infraestructura dio como resultado la formación de cuatro grupos (Silhouette de 0,662). En la Figura 1 se pueden ver los centroides de estos clústeres. Luego describimos cada uno de estos grupos usando la taxonomía de (Santoleri, 2013).

Figura 1. Gráfico centroides de TI de infraestructura

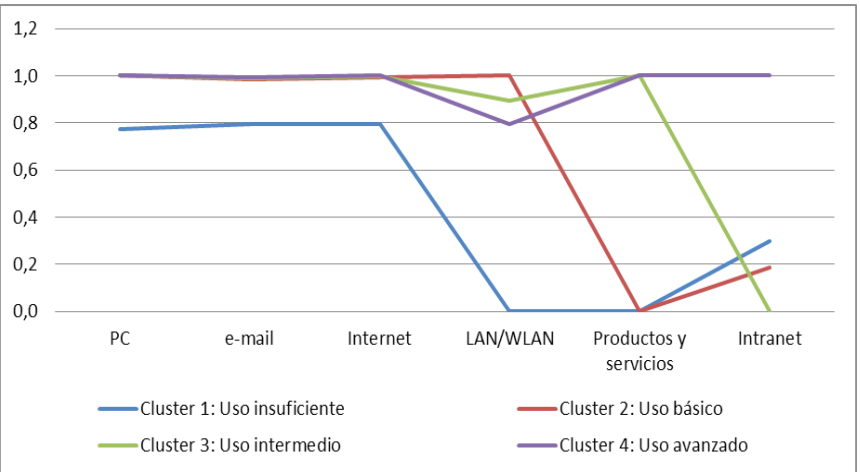

- Uso insuficiente (Clúster 1): Las compañías pertenecientes a este grupo son las que hacen menor uso de TI, dado que la mayoría de éstas sólo usa PC, e-mail e internet.

- Uso básico (Clúster 2): Todas las organizaciones de este clúster hacen uso de PC, e-mail, internet y de redes LAN/WLAN. Sin embargo, no utilizan intranet ni poseen un sitio web que permita conocer su empresa. 
- Uso intermedio (Clúster 3): Las empresas de este clúster hacen uso de PC, e-mail, internet, redes LAN/WLAN y poseen sitio web, el que permite conocer su empresa y los productos y/o servicios que ésta ofrece.

- Uso avanzado (Clúster 4): En este clúster se encuentran las empresas que hacen mayor uso de TI, dado que éstas utilizan prácticamente todas las tecnologías de infraestructura.

\section{2) TI transaccional}

En la Figura 2, se pueden ver los centroides de los conglomerados de las TI transaccionales, los cuales obtuvieron un índice Silhouette de 0,729 .

Figura 2. Gráfico centroides de TI transaccional.

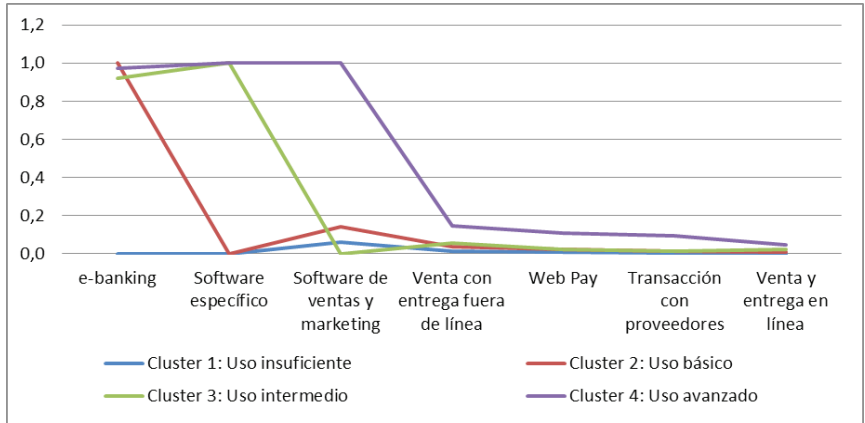

- Uso insuficiente (Clúster 1): Las empresas de este clúster no utilizan ninguna de las tecnologías listadas.

- Uso básico (Clúster 2): Las organizaciones de este conglomerado sólo hacen uso de e-banking.

- Uso intermedio (Clúster 3): Las firmas de este clúster únicamente usan software específico y e-banking.

- Uso avanzado (Clúster 4): En este clúster están las compañías que hacen mayor uso de TI transaccional, dado que todas usan e-banking, software específico y software de ventas y marketing.

\section{3) TI informacional - estratégica}

$\mathrm{Al}$ igual que sucedió con las TI anteriores, la clusterización de las variables de TI informacional - estratégica también dio como resultado la formación de cuatro conglomerados, los cuales obtuvieron un índice Silhouette de 0,899. No obstante, dos de los clúster formados sólo utilizan software de administración y software de logística y relación con proveedores, respectivamente, los cuales son tecnologías de similar complejidad de uso e implementación (Bansal \& Negi, 2008; Miyazaki, Idota, \& Miyoshi, 2012). Lo anterior obligó a que ambos clústeres se les asignara el mismo nivel de intensidad de uso. En la Figura 3 se muestran los gráficos de los centroides de las TI informacional - estratégica.
Figura 3. Gráfico centroides de TI informacional - estratégica.

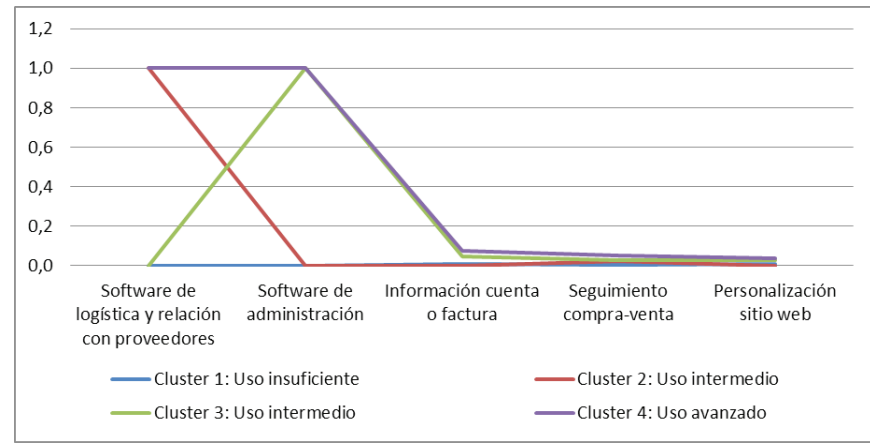

- Uso insuficiente de TI (Clúster 1): En este conglomerado están las firmas que no usan ningún tipo de estas de TI.

- Uso intermedio (Clúster 2 y Clúster 3): En este nivel de intensidad de uso se encuentran las empresas del clúster 2 y 3 que utilizan algún tipo de software para logística y relación con proveedores (clúster 2) o, alternativamente, poseen un software de administración (clúster 3).

- Uso avanzado (Clúster 4): Estas son las firmas que hacen mayor uso de TI informacional - estratégica, dado que todas utilizan software administración y software de logística y relación con proveedores.

Finalmente, la Tabla 5 muestra el número de Pymes existentes en cada nivel de intensidad de uso de los tres tipos de TI analizados en este trabajo.

Tabla 5. Número de Pymes por nivel de intensidad de uso.

\begin{tabular}{l|l|l|l|l}
\hline Tipos de TI & $\begin{array}{l}\text { Uso } \\
\text { insuficiente }\end{array}$ & $\begin{array}{l}\text { Uso } \\
\text { básico }\end{array}$ & $\begin{array}{l}\text { Uso } \\
\text { intermedio }\end{array}$ & $\begin{array}{l}\text { Uso } \\
\text { avanzado }\end{array}$ \\
\hline Infraestructura & 696 & 994 & 982 & 989 \\
\hline Transaccional & 636 & 2.049 & 646 & 330 \\
\hline $\begin{array}{l}\text { Informacional - } \\
\text { Estratégica }\end{array}$ & 1.341 & & 1.586 & 734 \\
\hline
\end{tabular}

\section{Resultados prueba de hipótesis}

1) H1: La intensidad de uso de TI influirá positivamente en el rendimiento organizacional de las Pymes.

$\mathrm{H} 1$ propone que la intensidad de uso de TI influirá positivamente en el rendimiento organizacional de las Pymes. Para comprobar esta hipótesis, se evaluó la correlación mediante Rho de Spearman, entre los tres tipos de TI y las variables de rendimiento planteadas. Los resultados de esta prueba estadística se muestran en la Tabla 6. 
Tabla 6. Tabla de correlaciones tipos de TI y variables de rendimiento organizacional.

\begin{tabular}{l|l|l|l|l|l|l|l|l}
\hline & Ganancias & Ventas & Costos & $\begin{array}{l}\text { Flujo de } \\
\text { caja }\end{array}$ & $\begin{array}{l}\text { Innovación } \\
\text { productos }\end{array}$ & $\begin{array}{l}\text { Innovación } \\
\text { logística }\end{array}$ & $\begin{array}{l}\text { Innovación } \\
\text { marketing }\end{array}$ & $\begin{array}{l}\text { Innovación } \\
\text { organizacional }\end{array}$ \\
\hline TI infraestructura & $0,233^{* *}$ & $0,447^{* *}$ & $0,397^{* *}$ & $0,297^{* *}$ & $0,194^{* *}$ & $0,159^{* *}$ & $0,121^{* *}$ & $0,162^{* *}$ \\
\hline TI transaccional & $0,173^{* *}$ & $0,400^{* *}$ & $0,356^{* *}$ & $0,270^{* *}$ & $0,163^{* *}$ & $0,112^{* *}$ & $0,069^{* *}$ & $0,141^{* *}$ \\
\hline TI informacional - estratégica & $0,225^{* *}$ & $0,543^{* *}$ & $0,512^{* *}$ & $0,339^{* *}$ & $0,177^{* *}$ & $0,171^{* *}$ & $0,131^{* *}$ & $0,144^{* *}$ \\
\hline
\end{tabular}

**. La correlación es significativa al nivel 0,01 .

La Tabla 6 muestra que los tres tipos de TI influyeron positivamente en las ganancias, ventas, flujo de caja e innovación empresarial de la mayoría de las industrias. Lo cual es consistente con parte de la literatura de TI encontrada. Por ejemplo (Santoleri, 2013) concluyó que la intensidad de uso de TI aumenta la capacidad innovadora de una organización, siendo esto demostrado por los tres tipos de TI estudiadas en este trabajo. También los resultados son consistentes con los de (Concha, Burr \& Suarez, 2014), el cual señaló que la mayoría de las Pymes percibieron que las TI aumentaron sus ventas. Asimismo, se corroboraron los resultados cualitativos de (Benavente, Lillo \& Turén, 2011), quienes concluyeron que entre propietarios y gerentes de empresas existía una percepción que las TI influían positivamente en las ventas y ganancias.

Un resultado interesante que es mostrado en la Tabla 6, es la relación entre intensidad de uso de TI y el aumento de costos. Lo que pareciera ir contra la percepción común sobre que las TI reducen los costos mediante la incorporación de procesos más eficientes. Sin embargo, estos resultados son concordantes con otros trabajos realizados que apuntan a que las TI introducen un aumento en los costos organizacionales (Andrade Loaiza, Tucull Gallardo \& González Gray., 2010; Benavente, Lillo \& Turén, 2011). Por lo tanto, el beneficio de las TI en las empresas se materializa a través de un incremento de las ventas, ganancias o mejoras en las capacidades de innovación de las compañías, pero implicaría un aumento de costos.
2) H2: El nivel de intensidad de uso de TI de la industria, moderará la relación entre la intensidad de uso de TI de las Pymes y su rendimiento organizacional.

Para comprobar esta hipótesis fue necesario, primero, establecer el nivel de intensidad uso de TI de todas las industrias de la muestra, la cual es mostrada en la Tabla 7. La Tabla 7 muestra que el nivel de intensidad de uso de las industrias en TI de infraestructura y transaccional presentó sólo dos estados: básico e intermedio. Las industrias que hicieron mayor uso de TI de infraestructura fueron la manufacturera (D), la de suministro de gas, electricidad y agua (E), la de comercio mayor y menor, reparación de vehículos, motocicletas, efectos personales y enseres domésticos $(\mathrm{G})$, la de transporte, almacenamiento y comunicaciones (I), la de intermediación financiera (J), la de actividades inmobiliarias, empresariales y alquiler $(\mathrm{K})$, y por último, la de otras actividades de servicios comunitarios, sociales y personales (O). Siendo esto consistente con (Gandhi, Khanna \& Ramaswamy, 2016), donde también se posicionaron a estas industrias como las más tecnologizadas en TI de infraestructura. En el caso de las TI transaccionales, las industrias E (suministro de gas, electricidad y agua) y J (intermediación financiera) fueron las que obtuvieron mayor nivel de intensidad de uso de TI transaccional. En cambio, en las TI informacional - estratégica, todas las industrias de la muestra hicieron uso intermedio de este tipo de tecnología.

Tabla 7. Nivel de intensidad de uso de TI por industria.

\begin{tabular}{l|l|l|l}
\hline Industria & TI infraestructura & TI transaccional & TI informacional - estratégica \\
\hline Agricultura, ganadería, caza y silvicultura (A) & Uso Básico & Uso Básico & Uso Intermedio \\
\hline Pesca (B) & Uso Básico & Uso Básico & Uso Intermedio \\
\hline Explotación de minas y canteras (C) & Uso Básico & Uso Básico & Uso Intermedio \\
\hline Industria manufacturera (D) & Uso Intermedio & Uso Básico & Uso Intermedio \\
\hline Suministro de electricidad, gas y agua (E) & Uso Intermedio & Uso Intermedio & Uso Intermedio \\
\hline Construcción (F) & Uso Básico & Uso Básico & Uso Intermedio \\
\hline $\begin{array}{l}\text { Comercio al por mayor y menor, reparación de vehículos automotores, mo- } \\
\text { tocicletas, efectos personales y enseres domésticos (G) }\end{array}$ & Uso Intermedio & Uso Básico & Uso Intermedio \\
\hline Hoteles y restaurantes (H) & Uso Básico & Uso Básico & Uso Intermedio \\
\hline Transporte, almacenamiento y comunicaciones (I) & Uso Intermedio & Uso Básico & Uso Intermedio \\
\hline Intermediación financiera (J) & Uso Intermedio & Uso Intermedio & Uso Intermedio \\
\hline Actividades Inmobiliarias, empresariales y de alquiler (K) & Uso Intermedio & Uso Básico & Uso Intermedio \\
\hline Otras actividades de servicios comunitarios, sociales y personales (O) & Uso Intermedio & Uso Básico & Uso Intermedio \\
\hline
\end{tabular}

En segundo lugar, se evaluó la relación, por industria, entre la intensidad de uso de TI de las empresas y las variables de rendimiento organizacional, tomando como variable moderadora el nivel de intensidad de uso de la industria. Sin embargo, este análisis sólo se limitó para las categorías de TI de infraestructura y transaccionales, dado que en la categoría de TI informacional - estratégica, todas las industrias de la muestra poseen el mismo nivel de intensidad de uso: Intermedio. Los resultados de estas correlaciones se exponen en la Tabla 8 (TI de infraestructura) y Tabla 9 (TI transaccionales). 
Por último, para validar $\mathrm{H} 2$ se supuso que: si existe un efecto moderador, los estadísticos de correlaciones deberían ser significativamente diferentes entre industrias que hacen uso básico de TI versus las que logran un uso intermedio. Por lo tanto, mediante T-test se compararon los $r$ promedios de las Tablas 8 y 9 , entre industrias de nivel básico e intermedio. El resultado de esta prueba se muestra en la Tabla 10.

Tabla 8. Tabla de correlaciones de TI de infraestructura por industria.

\begin{tabular}{|c|c|c|c|c|c|c|c|c|}
\hline \multicolumn{9}{|c|}{ Rho-Spearman promedio (TI Infraestructura) } \\
\hline Industria & Ganancias & Ventas & Costos & Flujo de caja & $\begin{array}{l}\text { Innovación } \\
\text { productos }\end{array}$ & $\begin{array}{l}\text { Innovación } \\
\text { logística }\end{array}$ & $\begin{array}{l}\text { Innovación } \\
\text { marketing }\end{array}$ & $\begin{array}{l}\text { Innovación } \\
\text { organizacional }\end{array}$ \\
\hline A & $0,182^{*}$ & $0,355^{\star *}$ & $0,347^{* *}$ & $0,186^{\star *}$ & $-0,025$ & $0,183^{*}$ & $0,171^{*}$ & $0,153^{*}$ \\
\hline $\mathrm{B}$ & $-0,072$ & $0,482^{* *}$ & $0,418^{\star *}$ & 0,126 & $0,271^{\star *}$ & $0,278^{\star *}$ & $0,229^{* *}$ & 0,115 \\
\hline $\mathrm{C}$ & $0,331^{* *}$ & $0,414^{* *}$ & $0,362^{* *}$ & $0,269^{* *}$ & 0,121 & 0,146 & 0,099 & 0,095 \\
\hline $\mathrm{D}$ & $0,176^{* *}$ & $0,492^{* *}$ & $0,491^{\star *}$ & $0,327^{* *}$ & $0,255^{\star *}$ & $0,158^{* *}$ & $0,138^{* *}$ & $0,126^{* *}$ \\
\hline G & $0,298^{* *}$ & $0,430^{* *}$ & $0,364^{* *}$ & $0,310^{* *}$ & $0,144^{\star *}$ & $0,168^{\star *}$ & $0,119^{* *}$ & $0,169^{\star *}$ \\
\hline $\mathrm{H}$ & 0,073 & $0,342^{\star *}$ & $0,294^{\star *}$ & $0,226^{\star *}$ & $0,191^{\star \star}$ & 0,072 & $0,165^{\star *}$ & $0,160^{\star *}$ \\
\hline $\mathrm{I}$ & $0,213^{\star *}$ & $0,496^{* *}$ & $0,491^{\star *}$ & $0,409^{* *}$ & $0,212^{\text {** }}$ & $0,212^{\star *}$ & 0,077 & $0,203^{\star *}$ \\
\hline $\mathrm{J}$ & 0,136 & 0,100 & 0,011 & 0,112 & $0,242^{\star *}$ & 0,112 & 0,124 & $0,192^{*}$ \\
\hline $\mathrm{K}$ & $0,222^{\star *}$ & $0,388^{* *}$ & $0,340^{* *}$ & $0,179^{* *}$ & $0,227^{\text {** }}$ & $0,129^{*}$ & 0,093 & $0,168^{\star *}$ \\
\hline
\end{tabular}

La Tabla 10 muestra que la relación entre la intensidad de uso de TI de infraestructura y todos los indicadores de rendimiento utilizados no es afectada por el tipo de industria a la que pertenece la Pyme (rechaza H2). En otras palabras, no existen diferencias estadísticamente significativas entre industrias que están en niveles básicos e intermedios de uso de este tipo de TI. Una posible explicación de lo anterior puede deberse a que este tipo de TI es de fácil acceso, dado que estas tecnologías poseen mayores índices de adopción en comparación a otros tipos de TI, principalmente por la continua caída que han tenido sus precios a lo largo de los años (Cette, Clerc, \& Bresson, 2015). Además, la principal utilidad de las TI de infraestructura es funcionar como base para futuras iniciativas o necesidades de negocio (Aral \& Weill, 2007), como por ejemplo, la utilización de otro tipo de tecnologías (TI transaccional o informacional - estratégica).

Tabla 9. Tabla de correlaciones de TI transaccional por industria.

\begin{tabular}{|c|c|c|c|c|c|c|c|c|}
\hline \multicolumn{9}{|c|}{ Rho-Spearman promedio (TI Transaccionales) } \\
\hline Industria & Ganancias & Ventas & Costos & Flujo de caja & $\begin{array}{l}\text { Innovación } \\
\text { productos }\end{array}$ & $\begin{array}{l}\text { Innovación } \\
\text { logística }\end{array}$ & $\begin{array}{l}\text { Innovación } \\
\text { marketing }\end{array}$ & $\begin{array}{l}\text { Innovación } \\
\text { organizacional }\end{array}$ \\
\hline B & $-0,086$ & $0,442^{* *}$ & $0,421^{\star *}$ & 0,071 & $0,242^{* *}$ & 0,072 & $0,189^{*}$ & 0,013 \\
\hline $\mathrm{C}$ & $0,200^{*}$ & $0,352^{* *}$ & $0,341^{* *}$ & $0,273^{* *}$ & $-0,005$ & $-0,098$ & 0,020 & 0,014 \\
\hline $\mathrm{D}$ & $0,093^{*}$ & $0,383^{* *}$ & $0,375^{* *}$ & $0,234^{* *}$ & $0,171^{* *}$ & $0,088^{*}$ & 0,009 & $0,140^{* *}$ \\
\hline E & 0,166 & 0,182 & 0,156 & $-0,060$ & $-0,014$ & $-0,082$ & 0,038 & 0,011 \\
\hline $\mathrm{H}$ & 0,077 & $0,402^{* *}$ & $0,334^{* *}$ & $0,265^{* *}$ & $0,240^{* *}$ & $0,118^{*}$ & $0,098^{*}$ & $0,170^{* *}$ \\
\hline I & $0,184^{* *}$ & $0,468^{* *}$ & $0,464^{* *}$ & $0,358^{* *}$ & $0,134^{*}$ & $0,183^{* *}$ & $0,119^{*}$ & $0,204^{* *}$ \\
\hline $\mathrm{J}$ & 0,172 & $0,301^{* *}$ & $0,211^{*}$ & $0,265^{* *}$ & 0,128 & 0,098 & 0,025 & $-0,079$ \\
\hline $\mathrm{K}$ & $0,199^{* *}$ & $0,333^{* *}$ & $0,285^{\star *}$ & $0,215^{* *}$ & $0,203^{* *}$ & 0,101 & $-0,007$ & $0,160^{* *}$ \\
\hline $\mathrm{O}$ & $-0,020$ & $0,492^{* *}$ & $0,365^{\star *}$ & $0,228^{* *}$ & 0,129 & 0,033 & $0,210^{* *}$ & $0,180^{* *}$ \\
\hline
\end{tabular}

Tabla 10. Comparación impacto de TI según nivel de intensidad de uso de la industria, mediante T-test.

\begin{tabular}{|c|c|c|c|c|c|c|}
\hline & \multicolumn{3}{|c|}{ TI infraestructura } & \multicolumn{3}{|c|}{ TI transaccional } \\
\hline & $\mathrm{t}$ & $\mathrm{gl}$ & Sig. & $\mathrm{T}$ & $\mathrm{gl}$ & Sig. \\
\hline Ganancias & $-0,604$ & 10 & 0,560 & $-0,882$ & 10 & 0,398 \\
\hline Ventas & 0,164 & 10 & 0,873 & $2,503^{*}$ & 10 & 0,031 \\
\hline Costos & 0,103 & 10 & 0,920 & $3,308^{*}$ & 10 & 0,008 \\
\hline Flujo de caja & $-0,564$ & 8,35 & 0,587 & 0,769 & 1,04 & 0,579 \\
\hline Innovación productos & $-0,337$ & 10 & 0,743 & 1,662 & 10 & 0,128 \\
\hline Innovación Logística & 1,231 & 10 & 0,246 & 1,262 & 10 & 0,235 \\
\hline Innovación Marketing & 1,328 & 4,65 & 0,246 & 1,071 & 10 & 0,309 \\
\hline Innovación Organizacional & $-1,256$ & 10 & 0,238 & $2,915^{\star}$ & 10 & 0,015 \\
\hline
\end{tabular}

ISSN: 0718-2724. (http://jotmi.org)

Journal of Technology Management \& Innovation @ Universidad Alberto Hurtado, Facultad de Economía y Negocios. 
Por lo tanto, a pesar de que existe una brecha tecnológica en TI de infraestructura entre las industrias, esta no representa un mayor efecto por dos razones. La primera es que las TI de infraestructura son de más fácil acceso y su penetración es más alta en comparación a otros tipos de tecnologías (Cette, Clerc \& Bresson, 2015). Segundo, la mayoría de las industrias que hace mayor uso de TI de infraestructura, no aprovecha la base que éstas otorgan para la adopción de nuevas tecnologías de carácter más avanzado, como lo son las TI transaccionales (Tabla 7).

En el caso de las TI transaccionales, el nivel de intensidad de uso de la industria si produce una diferencia en la relación entre uso de TI y rendimiento, sin embargo, este efecto es parcial y no se da para todos los indicadores de rendimiento usados (acepta parcialmente H2). Nuestros resultados muestran que el efecto de las TI transaccionales sobre las ventas, costos e innovación organizacional de las Pymes cambia, conforme el nivel de intensidad de uso de la industria a la cual las empresas pertenezcan. Siendo esto consecuencia de la brecha digital existente entre las industrias más y menos tecnologizadas, dado que el alto uso de este tipo de tecnologías influye directamente en el rendimiento de las Pymes (Aral \& Weill, 2007; Mora et al., 2012; Santoleri, 2013).

En resumen, H2 sólo se acepta parcialmente para las TI transaccionales, dado que los efectos de estas TI cambiaron según el nivel de intensidad de uso que tuviera la industria. Siendo esto consistente con lo planteado por (Cataldo \& McQueen, 2014).

\section{Conclusiones}

Nuestros resultados muestran que existe un efecto de las TI sobre el rendimiento de las Pymes. Además, para el caso de TI transaccionales, habría un efecto moderador del tipo de industria en esta relación. En ese sentido, el nivel de intensidad de uso de la industria modera el efecto de las TI transaccionales sobre el rendimiento organizacional de las Pymes; en particular, este efecto de moderación se observó sobre las ventas, costos e innovación organizacional. No obstante, los resultados no mostraron que la misma situación se presentara para el caso de las TI de infraestructura.

A modo de discusión, los resultados de la H1 (relación entre el uso de TI y rendimiento en Pyme), son consistentes con los de otros estudios (Benavente, Lillo \& Turén, 2011; Concha, Burr \& Suarez, 2014; Santoleri, 2013). Dado que los tres tipos de TI influyeron positivamente en las ganancias, ventas, flujo de caja e innovación de las Pymes de la muestra. Contra lo esperado, la intensidad de uso de estas TI se relacionó con un incremento en los costos. A pesar de lo contra-intuitivo de este resultado, también fue concordante con trabajos previos (Andrade Loaiza, Tucull Gallardo, González Garay, 2010; Benavente Lillo \& Turén, 2011).

En base a los resultados obtenidos, podemos dar respuesta a las dos preguntas planteadas al inicio de este trabajo. En la primera, nos preguntamos si toda combinación de TI otorga los mismos beneficios a las empresas, siendo esto desmentido por los resultados de H1, ya que las Pymes que hicieron mayor uso de TI son las que obtuvieron un mayor aumento en su rendimiento, en contraste a lo sucedido con las que hicieron un menor uso de TI. Siendo esto consistente con las conclusiones de trabajos previos (Brynjolfsson, 1993; Santoleri, 2013).

En la segunda, nos preguntamos si el grado promedio de uso de TI en la industria influye de forma importante en los efectos que puedan producir las TI dentro de las Pymes, lo cual es apoyado por los resultados de $\mathrm{H} 2$, dado que cuando existen brechas importantes de tecnologización en las industrias, el nivel de intensidad de uso de ésta alterará la relación entre la intensidad de uso de TI y el rendimiento de la Pyme, como se demostró con las TI transaccionales. Siendo esto consistente con lo señalado por (Cataldo \& McQueen, 2014).

Cabe mencionar que este estudio tiene algunas limitaciones. En primer lugar, dada la disponibilidad de los datos, no se pudieron incluir algunas variables de TI y rendimiento organizacional lo que podría haber extendido aun más los resultados encontrados. Esa limitación también afectó el tipo de clasificación que se utilizó para las TI, lo que obligó a unir las TI informacionales y estratégicas. Por último, este es un estudio cross-sectional por lo que sólo se considera información de un año, lo que impide comprobar cómo fueron cambiando los efectos de las TI sobre el rendimiento de las empresas con el paso del tiempo.

Sin embargo, las limitaciones de este estudio dan pie a trabajos futuros. Estos trabajos debiesen considerar nuevas variables e información longitudinal o de panel. Además, la inclusión de nuevas variables de TI permitiría estudiar más detalladamente los efectos de TI informacionales y estratégicas por separado.

\section{Referencias}

Abu-Jarad, I. Y., Yusof, N. A., \& Nikbin, D. (2010). A Review Paper on Organizational Culture and Organizational Performance. International Journal of Business and Social Science, 1(3), 26-46.

Ali, N., Habidin, N., Jamaludin, N., Khaidir, N., \& Shazali, N.. (2013). Customer Relationship Management and Performance in Malaysian Healthcare Industry. International Journal of Advancements in Research \& Technology, 2(1), 1-5.

Alves, M. F. R., Salvini, J. T. S., Bansi, A. C., Neto, E. G., \& Galina, S. V. R. (2016). Does the Size Matter for Dynamics Capabilities?: A Study on Absorptive Capacity. Journal of technology management \& innovation, 11(3), 84-93. doi:10.4067/S0718-27242016000300010

Andrade Loaiza, F., Tucull Gallardo, B., \& González Garay, G. (2010). Impacto que generan las tecnologías de la información y la comunicación (TIC’s) en las Pymes de la XII región.(Trabajo de Título). Universidad de Magallanes, Facultad de Ciencias Económicas y Jurídicas, Chile.

Aral, S., \& Weill, P. (2007). IT Assets, Organizational Capabilities, and Firm Performance: How Resource Allocations and Organizational Differences Explain Performance Variation. Organization Science, 18(5), 763-780. doi:10.1287/orsc.1070.0306 
Bannister, F., \& Remenyi, D. (2005). Why IT continues to matter: reflections on the strategic value of IT. The Electronic Journal of Information Systems Evaluation, 8(3), 159-168.

Bansal, V., \& Negi, T. (2008). A metric for ERP complexity. In Lecture Notes in Business Information Processing, 7, 369-379. doi:10.1007/9783-540-79396-0_32

Benavente, J. M., Lillo, N., \& Turén, J. (2011). ICT in Chilean firms. In ICT in Latin America - A microdata analysis (pp. 145-157). Santiago:ECLAC.

Bilbao-Osorio, B., Crotti, R., Dutta, S., \& Lanvin, B. (2014). The Networked Readiness Index 2014: Benchmarking ICT Uptake in a World of Big Data. In B. Bilbao-Osorio, S. Dutta, \& B. Lanvin (Eds.), The Global Information Technology Report 2014 - Rewards and Risk of Big Data (pp. 3-34). Geneva:World Economic Forum. Retrieved from http://reports.weforum.org/global-information-technologyreport-2014

Brasini, S., \& Freo, M. (2012). The impact of information and communication technologies: an insight at micro-level on one Italian region. Economics of Innovation and New Technology, 21(2), 107-123. doi:10.1080/10438599.2011.558175

Brynjolfsson, E. (1993). The Productivity Paradox of Information Technology: Review and Assessment. Communications of the ACM, 36(12), 66-77. doi:10.1145/163298.163309

Carr, N. G. (2003). IT doesn't matter. Harvard Business Review, 81(5), 41-49. doi:10.1109/EMR.2004.25006

Cataldo, A., \& McQueen, R. (2014). Strategic driver or unimportant commodity? A decade after "IT Doesn't Matter," SMEs prove that really does. Industrial Engineer, 46(2), 36-41.

Cette, G., Clerc, C., \& Bresson, L. (2015). Contribution of ICT Diffusion to Labour Productivity Growth: The United States, Canada, the Eurozone, and the United Kingdom, 1970-2013. International Productivity Monitor, 28, 81-88.

Chang, W., Park, J. E., \& Chaiy, S. (2010). How does CRM technology transform into organizational performance? A mediating role of marketing capability. Journal of Business Research, 63(8), 849-855. doi:10.1016/j.jbusres.2009.07.003

Concha, G., Burr, G., \& Suárez, P. (2014). Study on the Public Market Platform's Impact on SME in Chile. In International Conference on Theory and Practice of Electronic Governance, 435-441. doi:10.1145/2691195.2691211

Correa, C., \& Echavarría, G. (2013). Estimación del Aporte de las PyME a la Actividad en Chile, 2008 - 2011. Estudios Económicos Estadísticos - Banco Central de Chile, 101, 1-17. Retrieved from http:// www.bcentral.cl/-/estimacion-del-aporte-de-las-pyme-a-la-actividad-en-chile-2008-20-1
DeJarnett, L., Laskey, R., \& Trainor, H. E. (2004). From the CIO Point of View: The "IT Doesn't Matter" Debate. Communications of the Association for Information Systems, 13, 443-455.

Enjolras, M., Camargo, M., \& Schmitt, C. (2016). SMEs' Innovation and Export Capabilities: Identification and Characterization of a Common Space Using Data Spatialization. Journal of technology management \& innovation, 11(2), 56-69. doi:10.4067/s0718 27242016000200006

Fallahpour, S., Zadeh, M. H., \& Lakvan, E. N. (2014). Use of Clustering Approach For Portfolio Management. International SAMANM Journal of Finance and Accounting, 2(1), 115-136.

Gandhi, P., Khanna, S., \& Ramaswamy, S. (2016). Which Industries Are the Most Digital (and Why)?. Harvard Business Review. April issue. Boston, MA: Harvard Business School Publishing Corporation. Retrieved from https://hbr.org/2016/04/a-chart-that-shows-whichindustries-are-the-most-digital-and-why

Guzmán, G., Guzmán, M., \& Fuentes, R. (2016). Análisis del uso de las TIC en las pymes de Guayaquil en el año 2015. Oikos: Revista de la Escuela de Administración y Economía, 20(41), 109-119.

Hudson, M., Smart, A., \& Bourne, M. (2001). Theory and practice in SME performance measurement systems. International Journal of Operations \& Production Management, 21(8), 1096-1115. doi:10.1108/EUM0000000005587

Juárez, A. (2011). Uso de indicadores financieros para evaluar el impacto de las Prácticas de Alta Implicación. Working Papers on Operations Management, 2(2), 32-43. doi:10.4995/wpom.v2i2.847

Lohrke, F. T., McClure Franklin, G., \& Frownfelter-Lohrke, C. (2006). The Internet as an Information Conduit: A Transaction Cost Analysis Model of US SME Internet Use. International Small Business Journal, 24(2), 159-178. doi:10.1177/0266242606061838

Magal, S. R., Koslage, P., \& Levenburg, N. M. (2008). Towards a Stage Model for E-Business Adoption Among SMEs : Preliminary Results for Manufacturing and Service Firms. AMCIS 2008 Proceedings, 148, 1 - 13.

Miyazaki, S., Idota, H., \& Miyoshi, H. (2012). Corporate productivity and the stages of ICT development. Information Technology and Management, 13(1), 17-26. doi:10.1007/s10799-011-0108-3

Mora, M., Lerdon, J., Torralbo, L., Salazar, J., Boza, S., \& Vásquez, R. (2012). Definición de las brechas en el uso de las tic's para la innovación productiva en pymes del sector pecuario chileno. Journal of Technology Management and Innovation, 7(2), 171-182. doi:10.4067/ s0718-27242012000200014

Mortenson, M. J., \& Vidgen, R. (2016). A computational literature review of the technology acceptance model. International Journal of Information Management, 36(6), 1248-1259. doi:10.1016/j.ijinfomgt.2016.07.007 
Muñoz, L., \& Pitti, P. (2016). Generación y análisis de indicadores del uso de las TIC en PyMEs Agroindustriales. I+D Tecnológico, 12(2), 118-129.

Papastathopoulos, A., \& Beneki, C. (2010). Organizational forms based on information \& communication technologies (ICTs) adoption. Research in Business \& Economics Journal, 2, 1-18.

Pelleg, D., \& Moore, A. (2000). X-means: Extending K-means with Efficient Estimation of the Number of Clusters. 17th International Conference on Machine Learning, 727-734.

Ramírez-Correa, P., \& Alfaro-Pérez, J. (2011). El nivel de la inversión en tecnología de información no afecta el rendimiento empresarial: Evidencia empírica de las industrias manufactureras Chilenas. Journal of Technology Management and Innovation, 6(4), 225-241. doi:10.4067/s0718-27242011000400016

Reichert, F. M., \& Zawislak, P. A. (2014). Technological Capability and Firm Performance. Journal of Technology Management and Innovation, 9(4), 20-35. doi:10.4067/s0718-27242014000400002
Richard, P. J., Devinney, T. M., Yip, G. S., \& Johnson, G. (2008). Measuring Organizational Performance as a Dependent Variable: Towards Methodological Best Practice. Journal of Management, 35(3), 718-804. doi:10.2139/ssrn.814285

Rousseeuw, P. J. (1987). Silhouettes: A graphical aid to the interpretation and validation of cluster analysis. Journal of Computational and Applied Mathematics, 20, 53-65. doi:10.1016/03770427(87)90125-7

Santoleri, P. (2013). Diversity and Intensity of ICT use effects on product innovation : evidence from Chilean micro-data. 7 th Conference on Micro Evidence on Innovation and Development 24(6), 1-28. doi:1 $0.1080 / 10438599.2014 .946313$

Sheng, Y. P., \& Mykytyn Jr., P. P. (2002). Information Technology Investment and Firm Performance: A Perspective of Data Quality. 7th International Conference on Information Quality, 132-141.

Trung Pham, Q. (2010). Measuring The ICT Maturity Of SMEs. Journal of Knowledge Management Practice, 11(1), 1-12. 